\title{
Successful Drug Desensitization to Ustekinumab in a Patient with Crohn's Disease
}

\author{
Barrie Cohen $^{\mathrm{a}} \quad$ Gitit Tomer $^{\mathrm{b}}$ Tatyana Gavrilova ${ }^{\mathrm{a}, \mathrm{c}}$ \\ aDivision of Allergy and Immunology, Department of Pediatrics, Montefiore Medical \\ Center, Albert Einstein College of Medicine, Bronx, NY, USA; b Division of Gastroenterology, \\ Hepatology and Nutrition, The Children's Hospital at Montefiore, Albert Einstein College of \\ Medicine, Bronx, NY, USA; 'Division of Allergy and Immunology, Department of Medicine \\ and Pediatrics, Montefiore Medical Center, Albert Einstein College of Medicine, Bronx, NY, \\ USA
}

\section{Keywords}

Ustekinumab · Desensitization · Crohn's disease

\begin{abstract}
Ustekinumab is a monoclonal antibody used as treatment for various inflammatory conditions. We present a pediatric patient with Crohn's disease who did not tolerate infliximab and was then changed to ustekinumab. He developed anaphylaxis to the medication after the second dose. A drug desensitization protocol was created by the allergy team leading to successful administration of both intravenous and then subcutaneous ustekinumab. As monoclonal agents become mainstays of therapy for inflammatory conditions, there are increased reports of allergic reactions. Prior reports and protocols of ustekinumab desensitization have not been reported. This case report highlights successful desensitization to ustekinumab as well as the importance of a multidisciplinary approach to addressing treatment needs of patients who develop life-threatening reactions to such medications.
\end{abstract}

\section{Introduction}

Ustekinumab is a human monoclonal antibody against interleukins (IL)-12 and 23 used in chronic autoinflammatory conditions such as psoriasis and inflammatory bowel disease and is available in intravenous (IV) and subcutaneous (SC) forms. Ustekinumab has typically demonstrated a favorable safety profile although rare hypersensitivity reactions have been reported [1]. Here, we describe a patient who initially tolerated an IV dose and subsequently developed anaphylaxis to SC ustekinumab, followed by successful IV and SC desensitizations. 


\section{Case Presentation}

Written informed consent was obtained from the patient's parent to publish this case. A 10-year-old boy presented to allergy clinic after an allergic reaction to ustekinumab. He was diagnosed with Crohn's Disease (CD) (upper GI tract, small bowel, and ileocolonic involvement) 6 months prior to this visit. Previous therapies included steroids, infliximab, methotrexate, folic acid, and omeprazole. The infliximab level before the third infusion showed a level of $<1$ $\mu \mathrm{g} / \mathrm{mL}$ and elevated anti-infliximab antibodies of $48 \mathrm{U} / \mathrm{mL}$. Subsequently, methotrexate was added and the infusion frequency was decreased to every 4 weeks, during which the third and fourth infusions were well tolerated. However, after the fifth infliximab infusion, he complained of throat pruritus, difficulty breathing, and severe abdominal pain (although at this time, infliximab level was $27.1 \mu \mathrm{g} / \mathrm{mL}$ with no detectable antibodies). He was admitted to the hospital for observation and treated with antihistamines.

In addition to the allergic reaction, the patient had modest inflamamatory bowel disease (IBD) symptom improvement with infliximab. Therefore, a decision was made to change therapy to ustekinumab. The first dose of IV ustekinumab $260 \mathrm{mg}$ was well tolerated and after 8 weeks, the second dose of ustekinumab ( $90 \mathrm{mg} \mathrm{SC}$ ) was administered in the thigh. Approximately 15 min after the injection, he developed urticaria on his face, neck, and legs, severe abdominal pain, and vomiting. He was taken to the emergency department where epinephrine and antihistamines were administered with improvement after one hour. There was no hypotension or respiratory symptoms. He was admitted overnight for observation and awoke with hives in the morning, which resolved with antihistamines. He subsequently remained symptom-free and was discharged home. Of note, at the time of this reaction, he was completing a steroid taper of prednisone $10 \mathrm{mg}$ daily.

Given the previous allergic reaction to infliximab and now anaphylactic reaction to ustekinumab, the allergy and immunology service was consulted before switching yet again to another biologic. After a multidisciplinary discussion, ustekinumab was decided to be the optimal drug as he had already demonstrated clinical improvement after 2 doses. Therefore, our team designed a 12-step, three-bag protocol for IV ustekinumab desensitization which the patient tolerated successfully inpatient without additional premedication (shown in Table 1). Skin testing was not performed prior to the desensitization due to patient's anxiety. He continued the steroid taper during the desensitization.

Due to patient preference and ease of administration, we subsequently designed a SC desensitization protocol. Skin prick testing (SPT) with an undiluted formation and intradermal testing using a 1:10 dilution, similar to protocols for other biologic agents [2], were performed immediately prior to the SC desensitization, and both were negative. Based on negative skin testing and the number of injections required, we utilized a modified five-step SC desensitization protocol (shown in Table 1). He tolerated this desensitization in the outpatient infusion center without premedication and no adverse reaction. Eight weeks later, ustekinumab was successfully administered subcutaneously in the allergy and immunology outpatient clinic as a two step protocol with one-tenth of the dose administered followed by the remainder of the dose $30 \mathrm{~min}$ later.

\section{Discussion}

Biologic agents are becoming the mainstay of therapy for many immunologic disorders but the approach to hypersensitivity reactions to these agents still remains to be standardized. Studies suggest that low levels of drug antibodies can sometimes be overcome by dose escalation of anti-TNF therapy or addition of an immunomodulator [3]. This was the rationale for

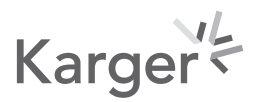


Table 1. IV and SC ustekinumab desensitization protocols

\begin{tabular}{|c|c|c|c|c|c|c|}
\hline & $\begin{array}{l}\text { Solution, } \mathrm{mg} / \mathrm{mL} \text {, } \\
\text { formulation }\end{array}$ & $\begin{array}{l}\text { Rate, } \mathrm{mL} / \mathrm{h} \text { for } \\
\mathrm{IV} \text { formulation }\end{array}$ & $\begin{array}{l}\text { Time, } \\
\text { min }\end{array}$ & $\begin{array}{l}\text { Volume, } \\
\text { mL administered }\end{array}$ & $\begin{array}{l}\text { Dose administered } \\
\text { with this step, mg }\end{array}$ & $\begin{array}{l}\text { Cumulative } \\
\text { dose, mg }\end{array}$ \\
\hline \multicolumn{7}{|c|}{ IV Ustekinumab Desensitization } \\
\hline 1 & $0.010 \mathrm{IV}$ & 2.5 & 15 & 0.63 & 0.0065 & 0.0065 \\
\hline 2 & $0.010 \mathrm{IV}$ & 5 & 15 & 1.25 & 0.0130 & 0.0195 \\
\hline 3 & $0.010 \mathrm{IV}$ & 10 & 15 & 2.50 & 0.0260 & 0.0455 \\
\hline 4 & $0.010 \mathrm{IV}$ & 20 & 15 & 5.00 & 0.0520 & 0.0975 \\
\hline 5 & 0.104 IV & 5 & 15 & 1.25 & 0.1300 & 0.2275 \\
\hline 6 & $0.104 \mathrm{IV}$ & 10 & 15 & 2.50 & 0.2600 & 0.4875 \\
\hline 7 & $0.104 \mathrm{IV}$ & 20 & 15 & 5.00 & 0.5200 & 1.0075 \\
\hline 8 & 0.104 IV & 40 & 15 & 10.00 & 1.0400 & 2.0475 \\
\hline 9 & $1.032 \mathrm{IV}$ & 10 & 15 & 2.50 & 2.5795 & 4.6270 \\
\hline 10 & $1.032 \mathrm{IV}$ & 20 & 15 & 5.00 & 5.1591 & 9.7861 \\
\hline 11 & $1.032 \mathrm{IV}$ & 40 & 15 & 10.00 & 10.3181 & 20.1042 \\
\hline 12 & $1.032 \mathrm{IV}$ & 80 & 174.375 & 232.50 & 239.8958 & 260.0000 \\
\hline \multicolumn{7}{|c|}{ SC Ustekinumab Desensitization $(90 \mathrm{mg} / \mathrm{mL})$} \\
\hline 1 & $90 \mathrm{SC}$ & $\mathrm{N} / \mathrm{A}$ & 0 & 0.05 & 4.5 & 4.5 \\
\hline 2 & $90 \mathrm{SC}$ & $\mathrm{N} / \mathrm{A}$ & 15 & 0.1 & 9 & 13.5 \\
\hline 3 & $90 \mathrm{SC}$ & $\mathrm{N} / \mathrm{A}$ & 15 & 0.2 & 18 & 31.5 \\
\hline 4 & $90 \mathrm{SC}$ & $\mathrm{N} / \mathrm{A}$ & 15 & 0.25 & 22.5 & 54 \\
\hline 5 & $90 \mathrm{SC}$ & $\mathrm{N} / \mathrm{A}$ & 15 & 0.4 & 36 & 90 \\
\hline
\end{tabular}

adding methotrexate and decreasing the frequency of infliximab infusions. Interestingly, the patient had a clinical reaction to infliximab after his fifth infusion, at which time he had a therapeutic level of infliximab but no detectable antibodies. While hypersensitivity reactions to biologic agents are rare, their frequency is increasing as more targeted monoclonal antibodies are developed and patients have multiple exposures to the drugs [4]. All efforts should be made to safely keep patients on their optimal therapy to treat their underlying disease appropriately, including rapid drug desensitization. To our knowledge, there are no existing protocols for ustekinumab desensitization in either IV or SC formulations.

While drug challenges can be considered in patients with negative SPT, it is the authors' position that if there is a convincing history for IgE-mediated reaction, a desensitization should be pursued despite the negative, currently nonstandardized, skin test. Here, we chose to perform a desensitization initially despite negative SPT based on the patient's reaction of anaphylaxis. The negative predictive value of negative skin testing for monoclonal antibodies is unknown [5], and in previous reports, only approximately half of patients with hypersensitivity reactions who ultimately required desensitization had positive skin testing to monoclonal antibodies [6]. However, in some cases, utilizing SPT can be useful for risk stratification [7].

In order to allow for flexibility, after successful desensitization in the intensive care unit or similar inpatient setting, additional desensitizations can be done outpatient [4], as was successfully tolerated in this case. The SC protocol used here was based on a modified version of a prior omalizumab desensitization [8] as well as several other rapid 
drug desensitization protocols of monoclonal antibodies [5]. Here, once the desensitizations were achieved, the patient tolerated routine SC treatments administered by the gastroenterology team as single dose injections. We expect him to able to avoid another desensitization to this agent as long as he does not miss a dose. He maintained a therapeutic level of ustekinumab and did not develop antibodies, though this was not checked at the time of the reaction.

Rapid drug desensitizations are most useful in type 1 hypersensitivity reactions, as seen in our patient, but can be used in a mixed type of reaction as well [7]. Prior to exploring alternative, second-line treatment options for patients' underlying diseases, consultation with an allergist is indicated to determine if the patient is a candidate for rapid drug desensitization. In conclusion, we report successful desensitization to ustekinumab after anaphylaxis in a patient with CD, in both IV and SC formulations.

\section{Statement of Ethics}

Ethics approval was not required by our institution as this is a single de-identified case report. Written consent from the patient/guardian in accordance with institution policy was obtained. Written informed consent was obtained by the patient's parent to publish this case.

\section{Conflict of Interest Statement}

The authors have no conflicts of interest to disclose.

\section{Funding Sources}

This manuscript did not receive any funding.

\section{Author Contributions}

Dr. Cohen and Dr. Gavrilova designed the desensitization protocol together while Dr. Tomer provided the gastroenterology management of the patient. This manuscript was written together, with Dr. Cohen and Dr. Gavrilova contributing the portions on drug desensitization and biologic drug reactions and Dr. Tomer providing expertise in CD management and the patient's clinical course from a gastroenterology perspective.

\section{References}

1 Ghosh S, Gensler LS, Yang Z, Gasink C, Chakravarty SD, Farahi K, et al. Ustekinumab safety in psoriasis, psoriatic arthritis, and Crohn's disease: an integrated analysis of phase II/III clinical development programs. Drug Saf. 2019;42(6):751-68.

2 Corominas M, Gastaminza G, Lobera T. Hypersensitivity reactions to biological drugs. J Investig Allergol Clin Immunol. 2014;24(4):212-25; quiz p. 1. following 25.

3 Kothari MM, Nguyen DL, Parekh NK. Strategies for overcoming anti-tumor necrosis factor drug antibodies in inflammatory bowel disease: case series and review of literature. World J Gastrointest Pharmacol Ther. 2017; 8(3):155-61.

4 Castells M. Rapid desensitization for hypersensitivity reactions to medications. Immunol Allergy Clin North Am. 2009;29(3):585-606.

\section{Karger'}


5 Bonamichi-Santos R, Castells M. Diagnoses and management of drug hypersensitivity and anaphylaxis in cancer and chronic inflammatory diseases: reactions to taxanes and monoclonal antibodies. Clin Rev Allergy Immunol. 2018;54(3):375-85.

6 Brennan PJ, Rodriguez Bouza T, Hsu FI, Sloane DE, Castells MC. Hypersensitivity reactions to mAbs: 105 desensitizations in 23 patients, from evaluation to treatment. J Allergy Clin Immunol. 2009;124(6):1259-66.

7 Yang BC, Castells M. Diagnosis and treatment of drug hypersensitivity reactions to biologicals: medical algorithm. Allergy. 2020;75(44).

8 Isabwe GAC, Garcia Neuer M, de las Vecillas Sanchez L, Lynch DM, Marquis K, Castells M. Hypersensitivity reactions to therapeutic monoclonal antibodies: Phenotypes and endotypes. J Allergy Clin Immunol. 2018;142(1): 159-e2. 